\title{
Herramientas tecnológicas en la enseñanza de las matemáticas durante la pandemia COVID-19
}

\section{Technological tools on mathematics teaching during a pandemic COVID-19}

\author{
Mg. Cynthia Iris Sánchez Pachas ${ }^{1}$ \\ https://orcid.org/0000-0003-1719-3518 \\ Universidad César Vallejo, Perú
}

Recibido: 10-04-2020

Aceptado: 20-08-2020

\section{Cita Recomendada}

Sánchez, C. (2020). Herramientas tecnológicas en la enseñanza de las matemáticas durante la pandemia COVID-19. Hamut'ay, 7 (2), 46-57.

http://dx.doi.org/10.21503/hamu.v7i2.2132

\section{RESUMEN}

La educación en nuestros días ha tenido un cambio vertiginoso, al pasar de la enseñanza presencial a la virtual debido al aislamiento generado por la pandemia del Covid-19, en este escenario los docentes han recurrido a las herramientas tecnológicas para el proceso de enseńanza - aprendizaje. Por lo que en este estudio documental se hace una revisión y análisis de la literatura, acerca de herramientas tecnológicas utilizadas en el área de matemáticas, con una temporalización de los últimos cinco años: 2016 - 2020, complementando con la experiencia de aula en estos primeros meses del ańo escolar 2020 con estudiantes del VII ciclo de la Educación Básica Regular.

Palabras clave: matemáticas, educación virtual, herramientas tecnológicas, competencias digitales.

\section{Abstract}

Education nowadays has had vertiginous changes in moving face to face to online teaching due to isolation generated by the COVID-19 pandemic, in this scenario teachers have resorted technological tools for teaching learning processes.

As far as a desk - study, is carried out a literature analysis and review, about technological tools used area of mathematics with timetable of the last five years: 2016-2020, supplementing with experience of classroom in these first months of the year 2020 with students of seventh cycle of regular basic education

Keywords: mathematics, virtual education, technological tools, digital competencies

\footnotetext{
1 Magister en Administración de la Educación, Licenciada en educación con especialidad en Matemática. Cursando estudios de Doctorado en Educación en la Universidad Cesar Vallejo. Correo: matematicasp@hotmail.com.
} 


\section{INTRODUCCIÓN}

Desde hace décadas la educación virtual era mirada con escepticismo, pero en la actualidad esta modalidad permite seguir brindando el servicio educativo en esta coyuntura debido a la pandemia de la COVID - 19. Siendo las matemáticas una de las áreas importantes a impartir con los estudiantes, ¿Cómo amoldar la enseñanza de las matemáticas en este contexto?, acción que en muchos escenarios sólo estaba centrada en el uso de un pizarrón y una tiza. Ya Monereo y Pozo (2001) expresaban que el estudiante del siglo XXI, recibe una formación del siglo XX, con maestros del siglo XIX. Aunado a ello, la enseñanza de las matemáticas siempre ha sido materia de estudio debido al reto que deben de afrontar los docentes para poder lograr aprendizajes significativos en los estudiantes y sobre todo mostrarles la utilidad que conlleva esta área en la vida cotidiana, fuera de otros factores que influyen en su aprendizaje evidentemente.

Pero la pandemia nos llegó, dando un giro abismal en el sector educativo, y el docente se ha visto obligado a adaptarse, y si además se considera que el enseñar matemáticas de modo presencial ya era un reto, hoy lo es aún más, debido a las circunstancias por las que se está pasando. La pandemia rompió el concepto físico de aula de clase y confrontó a los docentes a desarrollar o adquirir competencias digitales, porque se dio paso a la educación virtual, ya que hasta el momento es la única opción ante esta situación (Montero-Guerrero et al., 2020), pues queda claro que esta modalidad de educación ha permitido continuar con el servicio educativo no sólo en el Perú sino en el mundo (Lall, 2020; Daubney \& Fautley, 2020; Daniel 2020, Basilaia \& Kvavadze, 2020). A su vez, propició en el docente aflorar las competencias digitales, a reinventarse y sobre todo a usar la creatividad. En este contexto vemos que las herramientas tecnológicas por sí solas no generan aprendizaje, es el docente quien, a través de la planificación de sus sesiones de clase, hace uso de estas para poder obtener logros de aprendizaje (Viberg, 2020), teniendo que afrontar tres desafíos para este fin: Diseño de enseñanza (tareas, actividades y lecciones), su rol como docente y el contexto. (Drijvers, 2015).

Por ende, las herramientas digitales son un gran insumo para los docentes de matemáticas que permiten presentar de un modo distinto esta área curricular, ya sea en tiempo real o no, lo que permite el desarrollo de diversas habilidades, estilos y ritmos de aprendizaje para los estudiantes y al docente le sirve como un recurso innovador, ya que le permite generar metodologías activas y creativas (Alvites-Huamaní, 2017). Anteriormente, el manejo de estas herramientas eran usadas en gran medida por el grupo de docentes jóvenes o lo que serían los docentes Millenials (Hallman, 2017) maestros que pertenecen a este grupo generacional, pero el panorama que se muestra hoy es diverso, debido a la coyuntura los maestros han evidenciado adaptabilidad, siendo para muchos de ellos un nuevo aprendizaje, en el Perú la edad promedio de los maestros es de 45 años (MINEDU, 2019), el reto del maestro de educación secundaria es aún mayor debido a que son los adolescentes quienes tienen un manejo de las tecnologías. Por tal motivo, en un estudio realizado por Stein et al, (2020), se refuerza la importancia de la enseñanza del uso de estas herramientas en el proceso de formación docente en nuestros días. Pues como expresa Hallman (2017) el reto no sólo está en manejar tecnología sino saber enseñar con tecnología y eso aplica para todos quienes ejercen la docencia.

Por tanto, la enseñanza de la matemática inicia un nuevo proceso dentro de la virtualidad, el cual se verá apoyado en el uso de las herramientas tecnológicas, debemos ser conscientes que los estudiantes deben saber usarlas de manera adecuada y en favor de su aprendizaje; por lo cual la mediación del docente es vital.

\section{MÉTodo}

Al tratarse de un artículo de revisión descriptiva, se explora las diversas bases de datos como: Scopus, EBSCO, Google Académico, así como de páginas web relacionados a la temática a través de las palabras claves y operadores lógicos, teniendo en cuenta los últimos cinco años: 2016 al 2020. $\mathrm{Al}$ ser un tema incipiente, respecto a la coyuntura de la pandemia de la COVID - 19, las palabras de búsqueda en su mayoría han sido en inglés, dando lectura a cada una de ellas y seleccionando 
los que fundamentaban mejor la presente publicación. Como han sido: educación en tiempos de pandemia, mathematics teaching, digital tools in teaching mathematics y pandemic learning.

Al ser un estudio descriptivo documental no se va a presentar tablas ni figuras, sino se va a realizar un análisis desde la perspectiva de los autores que se refrendan en las referencias bibliográficas y en las citaciones realizadas, así como el exponer el uso de herramientas tecnológicas en el área de matemáticas desde la aplicación de estas como parte de la experiencia realizada dentro del aula de clase a cargo de la autora en el primer bimestre de clases impartidos en educación básica.

\section{Competencia digital docente}

Antes de la pandemia, en el Perú lo más cercano a trabajar con herramientas tecnológicas por los maestros de educación básica regular ha sido el uso de las laptops $\mathrm{XO}$, lo que formó parte del proyecto One Laptop per Child (OLPC), en nuestro país el promedio nacional de instituciones educativas en el nivel secundaria que poseen estas laptops son el 70,7\% (INEI, 2019) y junto a ellas existía el soporte del docente del aula de innovación. La enseñanza se impartía de modo presencial, por lo que la dinámica era totalmente distinta a la de nuestros días. Por tanto, ante esta crisis es que sale a la palestra la competencia digital docente, pues es gracias a esta situación que el maestro, en nuestro caso del área de matemática puede usar herramientas digitales para facilitar el aprendizaje en sus estudiantes. Pero ¿qué es una competencia digital?, para la UNESCO (2018) la competencia permite el uso, la aplicación y la gestión de dispositivos digitales a fin de intercambiar, comunicar contenidos que ayuden a dar solución a un problema en específico. El Marco Común de Competencia Digital Docente, (INTEFF, 2017) contempla cinco áreas para dicha competencia: Información y alfabetización informacional, comunicación y colaboración, creación de contenidos digitales, seguridad y resolución de problemas. Por tanto, el docente debe desarrollar estas áreas a fin de poder tener un óptimo desempeño. Pero la realidad nos muestra que aún hay vacíos respecto al conocimiento y manejo de herramientas digitales, tal es así que cuando se da inicio al periodo de aislamiento, el Ministerio de Educación (MINEDU), a través de su Portal Perú Educa permite el acceso a los cursos virtuales para todos los docentes del país, cuya temática gira en torno al uso de las tecnologías, con la finalidad que los docentes puedan desarrollar las competencias idóneas para poder apropiarse de las tecnologías, porque lo más importante es que los estudiantes continúen recibiendo educación. Esto muestra que los estados deben plantear nuevos modelos educativos, pues la pandemia exige un replanteamiento de las herramientas a utilizar en la educación virtual o a distancia. UNESCO (2020).

\section{El uso de las plataformas y el proceso de adapta- ción para la enseñanza de la matemática}

Al declararse en Perú la enseñanza virtual a razón del incremento de la enfermedad de la COVID-19, la pregunta que se hacían muchas instituciones educativas era ¿por dónde empezar? Las instituciones estatales trabajarían con la estrategia "Aprendo en Casa" emitido por el gobierno, pero las instituciones privadas y otra modalidad de gestión tendrían que hacer ajustes para poder brindar el servicio.

Esto llevó a realizar un diagnóstico inicial, por parte de los directivos de las instituciones educativas, con la finalidad de albergar a la mayor cantidad de estudiantes, indagar si había una computadora o laptop en casa, con accesorios que permitan acceder a una clase online, si los miembros de la familia iban a compartir los dispositivos electrónicos, ya que el teletrabajo se empezó a instaurar, o si el celular que poseían tenía las condiciones para poder acceder a las clases, como la memoria, a fin de que el estudiante pudiera albergar los archivos que se iban a emitir y buscar que estos sean livianos para evitar que esta se llene y lo más importante si contaban con una señal de internet.

Otro aspecto que surgió y se tuvo que realizar, era elegir las herramientas tecnológicas que permitan la enseñanza de las matemáticas en este contexto. Para lo cual se debía considerar el Currículo $\mathrm{Na}$ cional de Educación (CNEB), el cual contempla el desarrollo de cuatro competencias en el área de matemática en la Educación Básica Regular: i. Resuelve problemas de cantidad, ii. Resuelve pro- 
blemas de regularidad equivalencia y cambio, iii. Resuelve problemas de forma movimiento y localización y iv. Resuelve problemas de gestión de datos e incertidumbre que se apoyan en cuatro capacidades cada una y se sirven de los conocimientos matemáticos para tal logro. De acuerdo a la Resolución Viceministerial 093-2020-MINEDU, los docentes debían adaptar y/o priorizar las competencias a desarrollar si lo consideraban conveniente teniendo presente el contexto actual.

\section{Herramientas digitales en el área de matemática}

Las herramientas digitales apoyan a la enseñanza-aprendizaje del área de matemática proporcionando otras formas de poder transformar conceptos a través de la exploración y desarrollo de los mismos, aunque aún no se les ha dado todo el respaldo a estas herramientas, depende mucho del pensamiento que los docentes tengan frente a ellas. (Trurm \& Barzel, 2020). Pero en esta coyuntura, no es una opción dejar de usarlas, esto conlleva a la creatividad del maestro y a su pedagogía, pues es él quien diseña las actividades. Además, se debe tener en cuenta las habilidades digitales de los estudiantes evitando sobredimensionarlas, (Viberg, 2020) si bien es cierto se habla de nativos digitales en muchos casos ellos, solo son consumidores de contenidos, de redes sociales, y necesitan la orientación para poder usar de manera adecuada estas herramientas tecnológicas para su aprendizaje.

$\mathrm{Al}$ seleccionar estas herramientas tecnológicas se debe tener claro que estas ayuden al logro de aprendizaje del estudiante. Por lo que en este artículo en su mayoría están enfocadas a la competencia: Resuelve problemas de regularidad equivalencia y cambio, cuyos conocimientos están relacionados a álgebra, ya que no es fácil hacer tangible su aplicación como el resto de los contenidos.

Lo que se expone en la revisión de la literatura es una propuesta funcional, por lo que puede ser transferida a otras competencias. Por tal razón los criterios considerados para la selección de estas herramientas han estado ligados a la accesibilidad y funcionalidad, pues estas deberían de facilitar al estudiante su manejo, que permita la simulación: visualizar las gráficas o el movimiento de las mismas, crear propuestas tanto para el estudiante como para el docente, y además se ha considerado la interacción que tienen, debido a que el aprendizaje se construye mejor en pares o equipos, a través de colaboración, aquellos recursos que sean de fácil acceso para los estudiantes y que el docente domine las mismas sin generarle una carga adicional, y a su vez que permitan brindar y recibir información sobre sus avances para su evaluación y retroalimentación.

Después de haber evaluado diversas herramientas tecnológicas, se utilizaron las que se describen a continuación por cumplir con ciertas características mínimas y que podían ser asequibles, tanto para el docente como para los estudiantes, separando aquellas herramientas que se utilizan de manera asincrónicas de las sincrónicas.

\section{Herramientas Digitales asincrónicas:}

Debemos tener presente que en la parte asincrónica es donde el estudiante construye el conocimiento a través de la revisión de los insumos que brinde el docente, podemos hablar que el aprendizaje en los entornes virtuales está sustentado en el constructivismo (Hodam et.al, 2020, Jeong \& Akugizibwe, 2018), porque permite desarrollar un aprendizaje autónomo, siendo los estudiantes quienes manejan sus horarios y desarrollan las actividades con libertad donde los docentes asesoran a los estudiantes y monitorean su participación en cualquier momento del día (Daniel, 2020).

Por tanto, otra de las cuestiones para las instituciones educativas era como dar alcance de los contenidos a los estudiantes, por lo cual, se tuvo que buscar una alternativa que no implique trabajar una plataforma desde cero y que sea de fácil acceso para los estudiantes, por lo que a continuación se describirá herramientas utilizadas desde la experiencia de nuestras sesiones de clases.

Google Classroom (classroom.google.com). Esta plataforma que nació en el 2014 fue elegida porque permite compartir la información del docente, es de fácil manejo e intuitiva para el estudiante. (Saeed et al., 2018). Pues sólo basta con tener un usuario en Gmail y tiene acceso a todas las aplicaciones de Google (Alves et al, 2018), es gratuita y los beneficios son variados, era la primera vez 
que los estudiantes trabajaban con ella, no había forma de explicarles más que a través de tutoriales sobre su manejo y como debían enviar los trabajos. Además, los íconos ayudaban a clarificar si se trataba de un material o una tarea. Permite la interacción entre alumnos, profesor - alumno (os) que se complementa con los hangouts y el Gmail. (Bondarenko et al., 2019)

Para el desarrollo del área de matemática, el Classroom permitió compartir los archivos y videos que el docente podía elaborar, pero con la práctica era más fácil brindar el link que anexar el vínculo de Canal de Youtube que el docente había creado, porque recargaba el Drive y ponía lento el sistema. Una de las ventajas es que permite la visualización general de los temas de manera ordenada, pero como desventaja, no se puede visualizar si los estudiantes han revisado los materiales, así como poder cerrar el acceso de una tarea si ya se cumplió la fecha límite.

\begin{tabular}{|c|c|}
\hline SISTEMA DE INECUACIONES & \\
\hline (1) Mitos sobre las matemáticas y el cerebro. & Publicado 22 abr: \\
\hline (1) SELLOAdicional & Publicado. 22 abr. \\
\hline (1) trabajo enclase & Publicasdo. 22 abr. \\
\hline
\end{tabular}

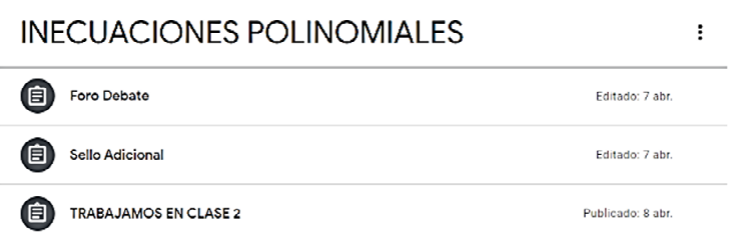

Figura 1. Interfaz del Google Classroom para el área de matemática.

Fuente: Elaboración propia (2020)

Hyperdocs (https://hyperdocs.co/). Ahora cuál era la forma más adecuada para poder presentar la información o ayudarles a seguir una ruta a los estudiantes. Los Hyperdocs fueron de gran ayuda, son lecciones digitales que están ordenadas y contienen todos lo hipervínculos en un solo documento que puede ser desde una diapositiva hasta un archivo de Word, cumplen la función de instrucción directa. (Carpenter, Trust \& Green, 2020). Por lo cual funciona como un instructivo, esto tiene un formato a seguir que puede ser adaptado, es una gran herramienta que fomenta el trabajo colaborativo tanto del docente como del estudiante. La ventaja es que todas las herramientas digitales están en un solo lugar permitiendo al estudiante centrarse en la información brindada.

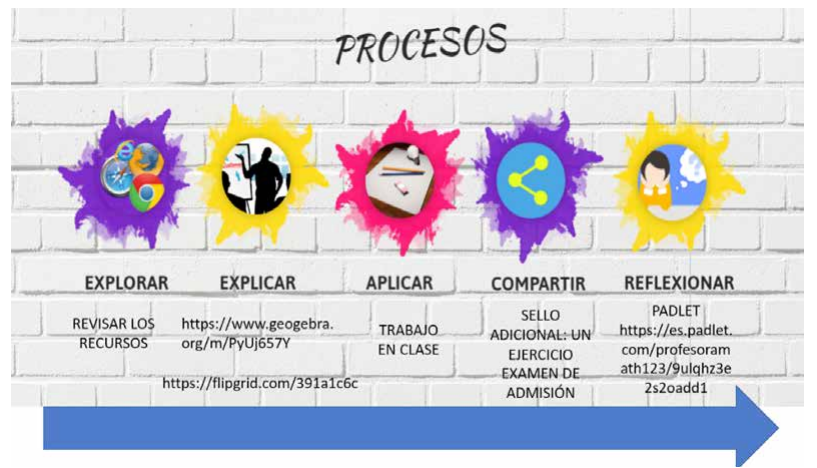

Figura 2. Modelo de aplicación del Hyperdoc para el tema de Productos Notables.

Fuente: Diapositiva elaboración propia (2020).

Foros. Una de las actividades que ayuda en el área de matemática son los foros, estas se realizan con los comentarios en clase, uno puede pensar que esto solo puede ayudar a las áreas de humanidades. En una sesión de Sistema de Ecuaciones se les planteó a los estudiantes una situación, ellos pudieron intercambiar ideas y tal vez se pudo pensar que la digitación de términos matemáticos pudo haber sido un impedimento, pero no fue así.

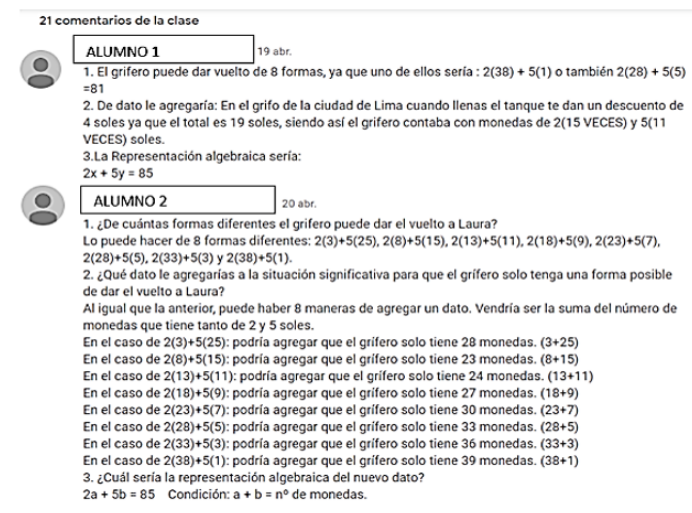

Figura 3. Intercambio de opiniones de los estudiantes en el foro en la sesión de sistema de ecuaciones en Classroom del área de matemática

Fuente: Elaboración propia (2020).

\section{Herramientas digitales sincrónicas}

En el área de matemática los alumnos necesitan despejar dudas e interrogantes, si bien es cierto pueden dejar los comentarios privados. La interacción en tiempo real, era necesaria. Es aquí donde las plataformas de videoconferencias que estaban destinadas al área de los negocios en su mayoría, que ayudaban a ahorrar tiempo y acortar distan- 
cias fueron el primer salvavidas que permitió la interacción en tiempo real mencionaremos a Zoom y Meet debido a que hubo una transición que se detalla a continuación:

Zoom (https://zoom.us/). Entre sus ventajas tenemos, que permite al docente tener mayor control de la clase, ya que él puede administrar el chat, puede enviar mensaje privados o general, permite compartir pantalla y facilita la proyección de vídeo. se puede aceptar a los estudiantes sin interrumpir la sesión, tiene complementos ya instalados que permiten expresar a los estudiantes sus emociones respecto a un tema, realizar votaciones, levantar la mano. Y lo más valorado en matemáticas, permite formar grupos en tiempo real, fomentando el trabajo colaborativo. Y posee una pizarra digital que es propia del programa. Además, se puede configurar la reunión con clave y dar algunos soportes de seguridad, se puede visualizar a todos los participantes. Pero tiene algunas desventajas que no permitían realizar algunas acciones, porque si no se tiene la cuenta corporativa, lo cual exige que la institución educativa cuente con una página web institucional, el tiempo de las sesiones es de 40 minutos, lo cuestionado es respecto al fenómeno llamado Zoombombing, que consiste en la interrupción de terceras personas ajenas a la reunión para incomodar a los asistentes. (Aiken, 2020), lo que ha llevado a la empresa dar pautas para proteger las aulas virtuales (Gallagher,2020).

Google meet (https://meet.google.com/). Como el tiempo de 40 minutos era insuficiente, se consideró por conveniente trabajar con el coloquialmente conocido como "meet". Este forma parte del paquete de las herramientas que brinda Google, fue recién el de 4 de mayo de 2020, que empezó a ser gratuita para todos los usuarios que tuvieran un correo en Gmail y este no fuera corporativo.

Las ventajas de este recurso es que es muy intuitivo, tiene conexión con el calendar de Google permitiendo agendar con anticipación las reuniones, además puedes agregar a los estudiantes si es que el docente trabaja con Google Classroom, permite albergar a 100 personas como máximo y el tiempo es ilimitado, por lo cual lo hace más atractivo, además, permite compartir pantalla y facilita la proyección de vídeo.
En cuanto a desventajas, es que no permite la grabación de la sesión, si es que no tienes una cuenta corporativa, hay que instalar complementos para poder hacer la interacción más amena, así como para tomar asistencia. Al dar acceso por primera vez a un estudiante este puede re-ingresar las veces que considere necesario, el compartir pantalla no permite visualizar el chat y debe de interrumpir la trasmisión para poder dar acceso a los que desean unirse a la reunión. Como no tiene una pizarra digital debe de usarse el Jamboard.

Jamboard (jamboard.google.com). Siempre es necesario detallar algunos procedimientos en el área de matemática y esta herramienta permite este proceso, pues es una pizarra digital, que permite el trabajo colaborativo. Se presentaba el ejercicio o la situación del día y los estudiantes expresaban sus respuestas a través de las notas adhesivas. Permite realizar una recolección de los saberes previos de un tema en específico. Lo más importante era que los estudiantes no perdieran tiempo en descargar otras apps, ya que también forma parte del paquete de Google, permitiendo que la sesión se desarrolle de manera fluida.

Genially (https://www.genial.ly/login). Desplaza al Power Point o al mismo Prezzi, pues esta herramienta permite brindar al alumnado la información teórica de una manera más amena e interactiva, al usar un recurso interactivo, este favorece el desarrollo de la atención y concentración, fomenta el interés por el tema y se puede proporcionar mayor cantidad de información. (Dzenskevich, 2020), sólo se necesita crear una cuenta con correo electrónico y posee plantillas para diversas presentaciones, infografías, entre otros. (Genially, 2019). Incluso se puede armar una sesión de clase insertando videos o programas sin necesidad de salir de la presentación.

Lo cual permite que la información llegue de un modo dinámico, es aquí donde el docente pone en evidencia su capacidad de diseñar a través de recursos tecnológicos adaptándolos a su contexto y realidad educativa, colaborar y usar recursos elaborados por otros especialistas. (Pepi, et.al, 2020)

Flipgrid (https://info.flipgrid.com/). Una de las capacidades dentro de cada una de las competencias es comunicar, por ejemplo, para la sesión de Productos Notables, los estudiantes tuvieron el reto 


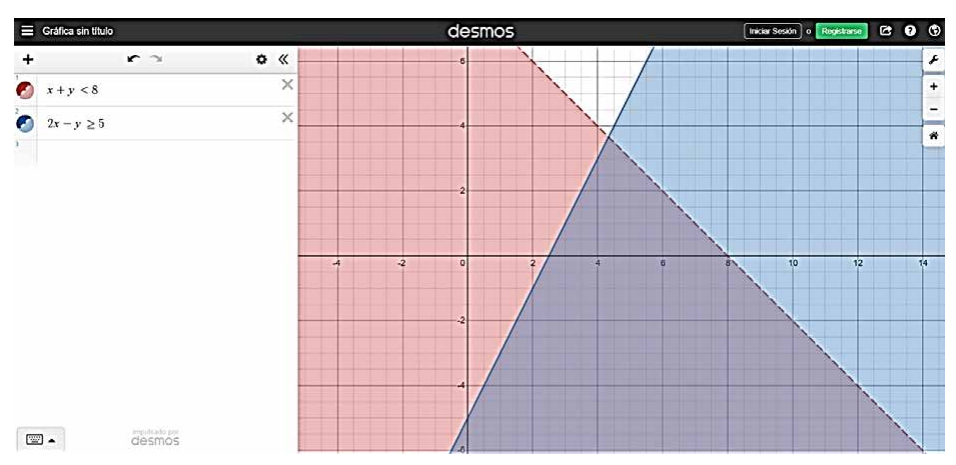

Figura 7. Interfaz de la calculadora gráfica Desmos, mostrando la solución de un sistema de inecuaciones.

Fuente: https://www.desmos.com/calculator?lang=es

der consultar de manera inmediata a su interrogante, en la mayoría de casos. Pero si tendrá al alcance los aportes que puede hacer el maestro respecto a sus avances. Así mismo, la evaluación tiene otro giro, rompió el esquema del docente que monitorea un examen en tiempo real por lo cual los estudiantes tienen libertad de poder hacer uso de toda clase de insumos que estén a su alcance. Por lo cual el docente del área de matemática debe buscar el equilibrio, a fin de obtener información relevante que sea provechosa a su práctica docente como para el estudiante.

Kaizena (https://www.kaizena.com/). Es otro de los complementos de Google. Las indicaciones por escrito no son suficiente para el estudiante, en matemáticas. Retroalimentación oral resulta efectiva si se realiza de manera personal. Esta herramienta permite enviar audios de voz, en la que las indicaciones pueden darse de manera detallada, pero para poder hacer uso de esta, tanto el estudiante como el docente deben descargar el aplicativo en la laptop.

Faster, Better

Feedback on Student

work

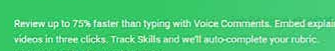

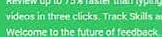

Figura 8. Interfaz para descargar Kaizena.

Fuente: Kaizena. com
Kahoot (https://create.kahoot.it/auth/login). Esta herramienta es una de las pioneras en el ámbito de evaluación en línea; tiene como estrategia al juego (Maraza et al, 2019) por ende, es del agrado de los estudiantes. Permite realizar la actividad tanto de manera sincrónica o asincrónica, y facilita la digitación de expresiones matemáticas. Lo beneficioso para el maestro es que te brinda información inmediata, menciona las preguntas donde los alumnos tuvieron dificultad, se puede visualizar la precisión con la que respondieron, permite fortalecer el uso de estrategias y procedimientos para resolver ejercicios.

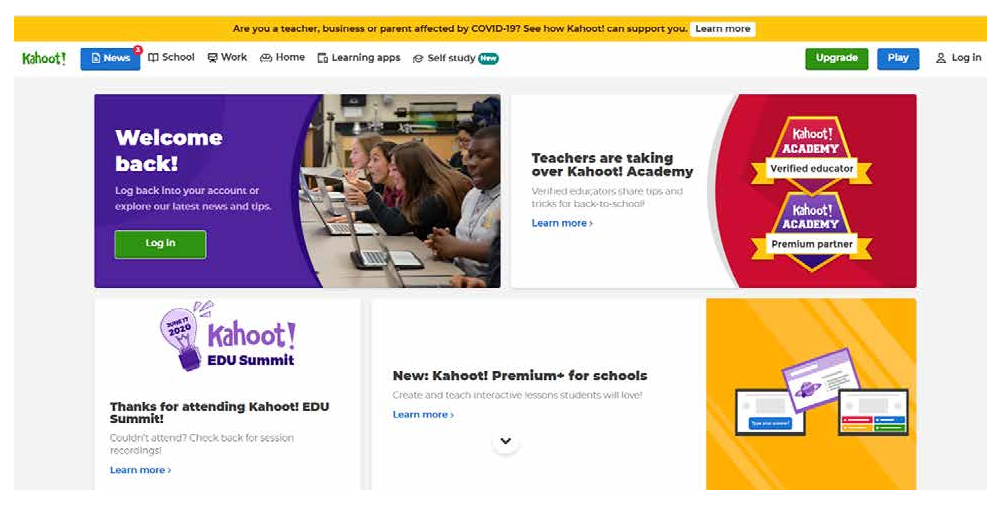

Figura 9. Interfaz de Kahoot

Fuente: Kahoot.com

Google Forms (https://docs.google.com/forms). Es crear un formulario de Google y transformarlo a cuestionario, brinda la puntuación a cada pregunta. Pueden ser preguntas de respuesta abierta o cerrada. Equivalen a una evaluación objetiva, pero esta debe tener sus restricciones y lineamientos a fin de recoger información que permita al docente reforzar al estudiante en lo que realmente necesita. Si bien hay que instalar el completo Forrm Limiter para poder dar un tiempo para el examen, la ventaja de tener la información en una hoja de Excel, permite identificar los intentos y la hora de entrega.

Si bien es cierto estas herramientas permiten obtener información de manera inmediata, debemos recordar que la finalidad de la evaluación es movilizar las cuatro capacidades para lograr la competencia, el contexto de la Pandemia ha traído consigo diversas temáticas que permiten generar experiencias de aprendizaje valiosas a los estudian- 
tes: Desde conocer las características del virus, la situación laboral en el país, los medicamentos utilizados por el momento para el tratamiento de la enfermedad, sobre la confección de implementos de protección hasta el crecimiento exponencial de los contagios, lo que ha conllevado a brindar a los estudiantes otra realidad en el ámbito educativo, que les permita comprender que las matemáticas van más de unos problemas en papel o ahora desde un ordenador, para que esta no sea concebida como una pérdida de tiempo sino direccionada a una realidad concreta, más vivencial (Orozco et al, 2019). La finalidad es que el estudiante sea capaz de revolver problemas que puedan suscitarse en la vida misma.

\section{Conclusiones}

Las herramientas digitales en esta coyuntura han facilitado el proceso de enseñanza de las matemáticas, siendo estas muy diversas, han propiciado en el docente el desarrollo de su creatividad e imaginación, evidenciando su competencia digital. Hay que tener en claro que las herramientas digitales son únicamente un insumo que ayuda al docente a lograr el aprendizaje, una herramienta al ser usada debe tener un fin pedagógico, pues el propósito es que el estudiante aprenda matemáticas.

Las matemáticas por su propia naturaleza no deben de eximirse del proceso: concreto, gráfico y abstracto. Las herramientas digitales deben ayudar a seguir ese proceso a fin que el estudiante pueda interiorizar y comprender el porqué de los temas en el área.

El contexto de la pandemia provee temas de aprendizaje que deben ser aprovechado por el docente, sobre todo si consideramos las tecnologías como una herramienta o recurso alternativo para hacer más interactivo el proceso de enseñanza aprendizaje en tiempos difíciles donde la virtualidad tiene una omnipotencia. Además, se debe enseñar una matemática que explique los fenómenos ocurridos en la realidad, como por ejemplo en este contexto del COVID - 19, los estudiantes saben que el incremento de los contagios, se expresa a través de un crecimiento exponencial, una simulación o proyección esta se convierte en un punto de partida para comprender el porqué del álgebra que muchas veces por su abstracción no es visible a los ojos de los estudiantes. Este tipo de situaciones en que los modelos matemáticos expresan una situación en concreto, propician el uso de nuevas metodologías o estrategias de aprendizaje basado en proyectos, (Purwaningsih, et al. ,2020), lo que puede evidenciar el logro de tareas de desempeño que va más allá de una aplicación algorítmica de fórmulas. Porque las matemáticas favorecen el desarrollo del pensamiento crítico, (Vásquez, 2020), dado que el estudiante al comprender situaciones y analizarlas debe tomar decisiones, sobre todo acciones, no sólo a beneficio personal sino para el bien común.

El docente también debe tener cuidado cuando aplica las TIC en no forzar llevar todo lo presencial a lo virtual, como menciona Ortiz (2020) no hablamos de una transcripción, pero sí de traducción debido al uso de nuevas herramientas sin descuidar la exigencia académica. No es lo mismo trabajar con adultos que con adolescentes frente al ordenador. La capacidad de síntesis para poder brindar la información es vital, es facilitar al estudiante el cimiento para que él pueda seguir profundizando.

Como lo expresan Trurm \& Barzel,(2020) el pensamiento que tenga el docente sobre si el uso de estas herramientas son útiles para la enseñanza de las matemáticas, es determinante, tal vez sea está la razón por la que aún persiste en llevar la enseñanza de la matemáticas como se hacía de manera presencial, pues para estos autores quien posee una creencia positiva frente al uso de estas herramientas hace un uso continuo de estas y las explora, mientras que quien posee todo lo contrario sólo ve en la tecnología un obstáculo, mencionando que los estudiantes pueden ser dependientes de esta, lo cual puede generar que no logren los aprendizajes propuestos. Sin duda, la actitud frente a los cambios tiene repercusión y más aún si hablamos del proceso de enseñanza-aprendizaje.

Esta coyuntura nos ha mostrado la fortaleza de las comunidades de aprendizaje (Viberg, 2020), a través de las redes, cada una de estas herramientas expuestas, comparten información y experiencias que enriquecen la labor pedagógica, ya sea a tra- 
vés de los webinars en sus redes sociales. Y es que, compartiendo con otros docentes, muchos de estos productos digitales son adaptados y mejorados, animándolos a realizar el suyo.

Otro de los aspectos que sale a relucir en esta experiencia es que los estudiantes necesitan de esa interacción con sus pares. Las videoconferencias con los estudiantes, fueron esenciales, permitió dilucidar interrogantes que surgieron en la etapa asincrónica. Queda patente que el ser humano necesita de esa relación con otros. Y más aún en adolescentes.

Las herramientas digitales en cuanto a evaluación, están en su mayoría diseñadas para brindar la retroalimentación respectiva, toma fuerza que si se indica a los estudiantes donde deben mejorar, el aprendizaje es más sólido y autorregulado. Permitiendo aprender de manera colaborativa y autónoma, pues el estudiante es quien puede verificar sus aciertos y fallos; y puede hacerlo en tiempo real o no con otros compañeros, esto fomenta su autonomía en el logro de su aprendizaje.

La transformación digital ya se ha dado en las instituciones educativas, los alumnos se desarrollan en un entorno digital elaborando productos acordes a su entorno, e interactúan en las redes sociales. Con esto se evidencia la urgencia de seguir trabajando las competencias transversales: se desenvuelven en entornos virtuales generados por las TIC y Gestionan su aprendizaje de manera autónoma.

En estos días para los estudiantes ya no está prohibido un celular en clase, pueden trabajar en equipos y pueden usar el buscador para poder investigar sobre algún tema en específico; generando debate sobre la autenticidad de la información, manejan su tiempo y se organizan para resolver las actividades propuestas en la plataforma.

Finalmente, en Perú el retorno a clases presenciales es un enigma aún; por lo que los docentes deberán seguir indagando, dialogando e investigando, haciendo uso de su capacidad autodidacta, y del aprendizaje por ensayo - error, sobre cuál es la mejor manera de poder enseñar en estos tiempos de pandemia, no existen recetas, pero sí el ímpetu para poder brindar lo mejor a los estudiantes. Por tanto, queda en el tintero de como plantear un nuevo modelo pedagógico que permita tomar lo avanzado en las aulas virtuales y no regresar al siglo XIX.

\section{REFERENCIAS BIBLIOGRÁFICAS}

Aiken, A. (2020). Zooming in on privacy concerns: Video app Zoom is surging in popularity. In our rush to stay connected, we need to make security checks and not reveal more than we think. Index on Censorship, 49(2), 24-27.

https://doi.org/10.1177/0306422020935792

Alves, F., \& Lima, D. (2018). Uso de la clasificación para el análisis y la minería de datos en la herramienta de enseñanza-aprendizaje Google Classroom. Nuevas Ideas en Informática Educativa, 4, 589-594. Recuperado de: http://www.tise. cl/Volumen14/TISE2018/589.pdf

Alvites - Huamaní, C. (2017) Herramientas TIC en el aprendizaje en el área de Matemática: Caso Escuela PopUp, Piura-Perú. Hamut'ay, 4 (1), 18-30. https://doi.org/10.21503/ hamu.v4i1.1393

Basilaia, G., \& Kvavadze, D. (2020). Transition to Online Education in Schools during a SARS-CoV-2 Coronavirus (COVID-19) Pandemic in Georgia. Pedagogical Research, 5(4), https://doi.org/10.29333/pr/7937

Bondarenko, O., Mantulenko, S., Pikilnyak, 囚. (2019): Google Classroom as a Tool of Support of Blended Learning for Geography Students. Recuperado de https://arxiv.org/ ftp/arxiv/papers/1902/1902.00775.pdf

Carpenter J., Trust T. \& Green T. (2020). Transformative instruction or old wine in new skins? Exploring how and why educators use HyperDocs, Computers \& Education (2020), https://doi.org/10.1016/j.compedu.2020.103979

Daniel, S.J.(2020) Education and the COVID-19 pandemic. Prospects

https://doi.org/10.1007/s11125-020-09464-3

Daubney, A., \& Fautley, M. (2020). Editorial Research: Music education in a time of pandemic. British Journal of Music Education, 37(2), 107-114.

https://doi.org/10.1017/S0265051720000133

Desmos (23 de junio de 2020). Desmos Activity Builder. (Archivo de video) Youtube: https://www.youtube.com/watch?time_continue $=41 \& \mathrm{v}=\mathrm{KxVPtQ} 3 \mathrm{jUr} 4 \&$ feature $=\mathrm{emb}$ title

Drijvers, P. (2015). Digital technology in mathematics education: Why it works (or doesn't). In Selected regular lectures from the 12th international congress on mathematical education (pp. 135-151)..

https://doi.org/10.1007/978-3-319-17187-6_8

Dzenskevich D., "Creating interactive posters on astronomy in the online services thinglink and genially". Meždunarodnyj naučno-issledovatel'skij žurnal (International Research 
Journal) No. 4 (94) Part 2, (2020): 59. Tue. 21. Apr. 2020. https://doi.org/10.23670/IRJ.2020.94.4.034

Gallaghe, R (27 de marzo de 2020). Prácticas recomendadas para proteger su aula virtual. Recuperado de: https://blog. zoom.us/es/practicas-recomendadas-para-proteger-su-aula-virtual/

Genially (2 de julio de 2019). ¿Qué es Genially? Descubre nuestro universo genial. (Archivo de video) Youtube:https:// www.youtube.com/watch?time_continue $=36 \& v=S F D x-$ gW8kuHg\&feature=emb_title

Hallman, H. (2016). Millennial teachers: Learning to teach in uncertain times. 2

https://doi.org/10.4324/9781315562582

Hodam, H.; Rienow, A.; Jürgens, C. (2020). Bringing Earth Observation to Schools with Digital Integrated Learning Environments. Remote Sens, 12, 345.

https://doi.org/10.3390/rs12030345

INEI (2019). Principales Resultados de la Encuesta Nacional a Instituciones Educativas de Nivel Inicial, Primaria y Secundaria, 2018. Recuperado de: https://www.inei.gob. pe/media/MenuRecursivo/publicaciones_digitales/Est/ Lib1684/libro.pdf

INTEF (2017). Marco Común de Competencia Digital Docente - Septiembre 2017.Recuperado de: https://aprende.intef.es/sites/default/files/2018-05/2017_1020_Marco-Com\%C3\%BAn-de-Competencia-Digital-Docente.pdf

Jeong Yong, A. \& Akugizibwe, E. (2018). An e-Learning Model for Teaching Mathematics on an Open Source Learning Platform. The International Review of Research in Open and Distributed Learning. https://doi.org/10.19173/ irrodl.v19i5.3733

Lall, Shatakshi \& Singh, Nardev. (2020). CoVid-19: Unmasking the new face of Education. International Journal of Research in Pharmaceutical Sciences. 11. 48-53..

https://doi.org/10.26452/ijrps.v11iSPL1.2122

Maraza, B., Cuadros, L., Fernandez, W., Alay, Y., Addison,A. (2019). Análisis de las herramientas de gamificación online Kahoot y Quizizz en el proceso de retroalimentación de aprendizajes de los estudiantes. Revista Referencia Pedagógica, 7(2): 339-362.0

MINEDU (2019) - Encuesta Nacional a Docentes de Instituciones Educativas Públicas y Privadas (ENDO), 2018. Recuperado de: http://escale.minedu.gob.pe/uee/-/document_library_display/GMv7/view/5384052

Mohmmed, A.O., Khidhir, B.A., Nazeer, A. \& Vijayan, V. (2020) Emergency remote teaching during Coronavirus pandemic: the current trend and future directive at Middle East College Oman. Innov. Infrastruct. Solut. 5, 72. https:// doi.org/10.1007/s41062-020-00326-7

Moreno-Guerrero, A.-J.; Aznar-Díaz, I.; Cáceres-Reche, P.; Alonso-García, S. E. (2020). Learning in the Teaching of Mathematics: An Educational Experience in Adult High School. Mathematics 2020, 8, 840. https://doi.org/10.3390/math8050840

Orozco, M., Obredor, S., Mejía, F. (2019). Importance of coupling the teaching methodologies of mathematics with the learning styles of digital natives. V International Conference Days of Applied Mathematics. Journal of Physics: Conference Series 1414 https://doi.org/10.1088/1742-6596/1414/1/012004

Ortiz, P. (2020). Teaching in the time of COVID-19. Biochemistry \& Molecular Biology Education, April 2020. https://doi.org/10.1002/bmb.21348

Pepin, B., Gueudet, G. \& Trouche, L. (2017). Refining teacher design capacity: Mathematics teachers' interactions with digital curriculum resources. ZDM Mathematics Education 49, 799-812.

https://doi.org/10.1007/s11858-017-0870-8

Pozo, J. y Monereo, C. (2001). ¿En qué siglo vive la escuela? El reto de la nueva cultura educativa. Cuadernos de Pedagogía. Número 298.

Purwaningsih, E. \& Sari, A. \& Yuliati, L. \& Masjkur, K. \& Kurniawan, B. \& Zahiri, M. (2020). Improving the problem-solving skills through the development of teaching materials with STEM-PjBL (science, technology, engineering, and mathematics-project based learning) model integrated with TPACK (technological pedagogical content knowledge). Journal of Physics: Conference Series. https:// doi.org/10.1088/1742-6596/1481/1/012133

Saeed, R. \& Al-Emran, M. (2018). Students Acceptance of Google Classroom: An Exploratory Study using PLS-SEM Approach. International Journal of Emerging Technologies in Learning (iJET). 13. 112-123. .

https://doi.org/10.3991/ijet.v13i06.8275

Stein, H., Gurevich, I. \& Gorev, D. (2020). Integration of technology by novice mathematics teachers - what facilitates such integration and what makes it difficult?. Educ Inf Technol 25, 141-161.

https://doi.org/10.1007/s10639-019-09950-y

Thurm, D., \& Barzel, B. (2020). Effects of a professional development program for teaching mathematics with technology on teachers' beliefs, self-efficacy and practices. ZDM Mathematics Education. https://doi.org/10.1007/s11858020-01158-6

https://doi.org/10.1007/s11858-020-01158-6

UNESCO (1 de abril de 2020). Aprendiendo en casa: educación a distancia para todos. Recuperado de https://es.unesco.org/news/aprendiendo-casa-educacion-distancia-todos

UNESCO (15 de marzo de 2018). Las competencias digitales son esenciales para el empleo y la inclusión social. Recuperado de https://es.unesco.org/news/competencias-digitales-son-esenciales-empleo-y-inclusion-social

Vadillo, J. (15 Marzo de 2020). Entrevista a Hugo Diaz. Hugo Díaz: "El desafío educativo del 2021 será combinar la educación presencial con la educación a distancia”. El Peruano. Recuperado de https://www.elperuano.pe/noticia-hugo-diaz-desafio-educativo-del-2021-sera-combinar-educa- 
cion-presencial-con-educacion-a-distancia-95876.aspx

Vásquez, C., Ruz, F. y Martínez, M. (2020). Recursos virtuales para la enseńanza de la estadística y la probabilidad: un aporte para la priorización curricular chilena frente a la pandemia de la COVID-19. TANGRAM - Revista de Educação Matemática. 3. 159-183.

https://doi.org/10.30612/tangram.v3i2.12299

Viberg, O. , Grönlund, A.\& Andersson,A. (2020): Integrating digital technology in mathematics education: a Swedish case study, Interactive Learning Environments, https://doi. org/10.1080/10494820.2020.1770801 